\title{
REGISTRO HISTORICO DE ANTECEDENTES VOLCÁNICOS Y SÍSMICOS EN LA PATAGONIA AUSTRAL Y LA TIERRA DEL FUEGO
}

MATEO MARTINIC B.

\section{RESUMEN}

Se presenta un registro actualizado sobre la actividad volcánica y los movimientos sísmicos de los que hay memoria histórica, desde la más remota antigüedad hasta el presente, a base de información documental (testimonios, relaciones, cartografía) y los datos aportados por la arqueología y los estudios paleoambientales. Se afirma la vigencia de un distrito volcánico-tectónico singular cuya actividad aperiódica merece ser conocida y considerada por razones de prevención de carácter social y económico.

PALABRAS CLAVES: Patagonia austral, Tierra del Fuego, volcanismo, sismicidad.

\section{HISTORIC RECORD OF VOLCANIC AND SEISMIC PRECEDENTS IN SOUTHERN PATAGONIA AND TIERRA DEL FUEGO}

\begin{abstract}
An updated record of the volcanic activity and seismic movements of known historic memory is presented, from the earliest antiquity till present, based on documental information and data contributed by archaeology and palaeoenvironmental studies. The force of a singular volcanic-tectonic district is affirmed, whose not periodic activity deserves to be known and considered for prevention reasons of social and economic character.
\end{abstract}

KEY WORDS: Southern Patagonia, Tierra del Fuego, volcanism, seismicity.

\section{INTRODUCCIÓN}

El interés del ambiente académico respecto de la actividad sísmica y volcánica en la Patagonia austral y la Tierra del Fuego es asunto de reciente data. Ha contribuido a ello la rara ocurrencia de estos fenómenos telúricos en un país como Chile en el que, por el contrario, desde Arica a Chiloé (dos tercios del territorio americano de la República) los mismos se han sucedido desde que hay

\footnotetext{
Profesor Emérito, Centro de Estudios del Hombre Austral, Instituto de la Patagonia, Universidad de Magallanes, Chile. Correo electrónico: magallania@umag.cl
} 
memoria y hasta el presente con una frecuencia, y a veces también violencia, que permiten caracterizarlo como uno de los países con mayor actividad sísmica y volcánica de la Tierra. Otra razón de este insuficiente conocimiento (o virtual ignorancia para muchos) reside en el hecho de haber ocurrido tales fenómenos en sectores andinos por lo general de difícil o imposible acceso, y que, además, o están deshabitados o cuentan con escasa población. Sólo las erupciones del volcán Hudson en 1991, y la más reciente del volcán Chaitén durante este año, así como los movimientos sísmicos de prolongada vigencia en el fiordo Aysén (2007), en todos los casos con consecuencias catastróficas y que por lo mismo, recibieron una amplia cobertura en los medios noticiosos, han despertado el interés del ambiente académico y del común de la gente, incluyendo a las autoridades gubernativas nacionales y locales.

Pero, no cabe duda, tanto el fenómeno volcánico, como el sísmico, se han manifestado recurrente aunque aperiódicamente a lo largo del tiempo desde el Pleistoceno Final hasta el presente, según ha podido constatarse a través del estudio de sedimentos de cenizas en diferentes yacimientos, en lo referido a las épocas más antiguas, esto es, a las anteriores al siglo XVI, y del registro histórico documental y testimonial para la época reciente, en particular desde el siglo XVIII en adelante. Por eso tratamos sobre la actividad volcánica histórica, considerando la "antigua" como perteneciente a lo más remoto hasta el siglo XVII, cuya información se basa en los estudios y registros sedimentarios y la "reciente", basada en antecedentes testimoniales y documentales.

Personalmente nos hemos ocupado reiteradamente sobre la materia (Martinic 1960 a y b, 1982, 1985, 1988 a y b) por lo que consideramos que puede ser de utilidad presentar todos los antecedentes históricos que han podido compulsarse acerca de los fenómenos volcánicos y sísmicos, debidamente actualizados, teniendo en vista la importancia que los mismos representan para los humanos y la naturaleza, y el riesgo serio que puede implicar, bajo diferentes respectos, su minusvaloración o desconocimiento, en la perspectiva posible de eventuales repeticiones.

\section{I.- ACTIVIDAD VOLCANICA}

Respecto de este fenómeno las fuentes informativas son varias y se consideran por separado.

\section{1.- La cartografía histórica}

Los primeros mapas nacionales con un grado apreciable de detalle e información geográfica fidedigna fueron elaborados durante la primera mitad del siglo XVII. Los mismos respondían al estado del conocimiento que se poseía en la época acerca de las características geográficas, naturales y sociales del entonces conocido como Reino de Chile, al cabo de un siglo de la presencia y asentamiento hispanos en el territorio. Expresión cabal de esa etapa de la cartografía nacional fue el mapa Tabula Geographica Regni Chile, del historiador jesuita P. Alonso de Ovalle, del que se conocen cuatro versiones publicadas entre 1646 y 1728 (Martinic, 1997). Todas ellas ofrecen una representación de la cordillera de los Andes, en tanto que eje vertebral del territorio, con el dibujo de montañas coniformes característico del arte cartográfico propio de la época, de las que dieciséis muestran una columna de humo para señalar su condición de volcanes activos. El más austral de los mismos se sitúa enfrentando la isla Madre de Dios, con el nombre de volcán de San Clemente. El siguiente hacia el norte frente a la isla de Chiloé. Importa señalar que la fuente principal del mapa de Ovalle fue la carta de Chile elaborada por Fray Gregorio de León en época anterior indeterminada y que se halla perdida desde hace siglos. De la misma, pues, pudieron derivar los antecedentes sobre una pretendida -o real- actividad volcánica en los Andes Patagónicos meridionales.

Esta mención que, de partida, lleva a pensar que se trata de la primera representación de un centro volcánico en los Andes de la Patagonia austral, pierde consistencia al recurrirse a la descripción pertinente del texto de la obra de Ovalle, donde, en lo que interesa, se precisa: [...] últimamente se ven dos, el uno que llaman Sin Nombre y está en cuarenta y cuatro [grados] escasos, y el de $S$. Clemente, que está en cuarenta y cinco y medio. Estos son los volcanes que se saben y se han visto en Chile y no tenemos noticia de otros que habrá hasta la Tierra del Fuego, porque hasta ahora no se ha penetrado tan adelante. ¿Quién duda que 
los haya? ${ }^{1}$. En efecto, la isla Madre de Dios utilizada como referente latitudinal en el mapa se enfrenta en general a los 50S, en evidente discordancia con la referencia del texto. De allí que cabe desestimar la situación más meridional y validarla para la latitud $44^{\circ} 30^{\prime}$ S. No obstante, otras cartas geográficas que se inspiraron en el mapa de Ovalle conservaron en la posición original (frente a Madre de Dios) la figura de un cono volcánico activo (Le Chili, de Nicolás Sanson D'Abbeville, 1656, y Destroict de Magellan, Terre et Isles Magellaniques,1658; La Terre et les Isles Magellaniques, de Guillaume Sanson, 1668). Más notable es la mención contenida en el Mapa Geographico de la América Meridional, de Juan de la Cruz Cano y Olmedilla (1775), hacia los 51을 de latitud, figurada con un volcán en erupción y el nombre V. de los Gigantes.

Cabe preguntarse si en aquella persistencia cartográfica (los Sanson) y en esta última pieza se dio una suerte de premonición geográfica que respondía a la pregunta del Padre Ovalle (¿Quién duda que los haya?). En el caso del mapa de Cano y Olmedilla procede la conjetura de si se trató de una mera repetición de la información del historiador jesuita o si, quizá, la figura y su denominación correspondieron a una noticia diferente de ulterior data, desconocida para la posteridad, que pudo emanar de otro distinto informante. Hay que convenir, en cualquier caso, en la originalidad del nombre que conforma una referencia implícita a los habitantes autóctonos, los patagones o gigantes de la antigua cartografía. En este respecto, recordamos haber visto hace años en la pared de una librería de Buenos Aires, un mapa de la Patagonia que tenía una mención a un volcán muy notoria, que lo situaba en la zona andino-marítima de Ultima Esperanza. Sensiblemente no pudimos retener el nombre del autor, ni la denominación de la pieza y la fecha de publicación. Que no fue una rareza tal lo confirma su reiteración en otra pieza cartográfica de ese tiempo, A New Map of South America, de John Cary (Londres, 1807).

En todos estos casos, desde Ovalle hasta Cary, debe aceptarse que la o las informaciones procedieron de referencias o testimonios que en su hora se tuvieron por fidedignos y como tales se incorporaron a los mapas. En cuanto a la Tierra del

1 Alonso de Ovalle, Histórica Relación del Reino de Chile (Instituto de Literatura Chilena, Santiago, 1969), pág. 43.
Fuego se refiere, de 1764 data la Carta Espherica del Remate de la América Meridional, de autor español desconocido, que muestra aproximadamente sobre la actual bahía Nassau la figura de una montaña humeante con la leyenda Bolcán de San Clemente. La referencia corresponde al avistamiento hecho el 24 de noviembre de 1712 por el capitán Josselin Gardin, mientras pasaba con el navío Saint Clement a la cuadra de la isla Hermite, ocasión en que observó y dejó constancia de una erupción volcánica hacia el interior del archipiélago fueguino. Idénticas o muy parecidas menciones se contienen en la Carta Reducida de la América Meridional de Amadeo Frezier (1772) y en el mapa de los estrechos de Magallanes y Le Maire construido por M. De Laborde y publicado en 1790.

\section{2.- La memoria indígena}

Es otra fuente informativa de gran interés sobre la materia, pues la misma refleja el conocimiento que tuvieron algunos pueblos aborígenes australes, en particular los aónikenk, en lo concerniente a los fenómenos de la naturaleza en su territorio. En efecto, su acabado dominio del mismo incluía la noción de las formas volcánicas que se observan en distintas zonas del interior, así como de movimientos telúricos ocasionales, acompañados o no por ruidos subterráneos y lluvias de cenizas, todo lo cual sería conservado por la memoria indígena. Viajeros del siglo XIX como Teófilo Schmid, George Musters, Francisco P. Moreno, Carlos M. Moyano y Ramón Lista, recogieron referencias ora generales ora específicas sobre las actividades telúricas y volcánicas que se registraban ocasionalmente en su territorio ancestral.

El explorador Musters que en su larga travesía de un año en compañía de los aónikenk pudo comprobar reiteradamente el buen conocimiento que los mismos tenían de su territorio, relata su propia impresión al pasar por los campos de lava de Pali Aike en abril de 1869, imaginando como pudo ser [...] cuando los volcanes estaban en actividad y vomitaban torrentes de lava y granizadas de rocas, en un período tal vez no muy remoto, agregando a continuación que más tarde, [...] en Santa Cruz, Casimiro me habló de un volcán en actividad que se encontraba a una distancia y en una dirección tales que lo hacían aparecer como perteneciente a esa cadena. Las vecindades de 
esta habían sido frecuentadas anteriormente por los indios, porque los guanacos solían refugiarse allí en gran número durante el invierno; pero la mayor parte de los caballos había muerto, envenenados por el agua de un arroyo próximo a la sierra, y poco después todos los toldos habían sido volteados por un terremoto, o por la vibración de una erupción, y desde entonces los indios no se aventuraban a acercarse a ese lugar. Importa señalar que la primera referencia de Musters corresponde a la cadena de cerros bajos donde destaca el cerro Diablo, posiblemente el de más reciente actividad (Skewes, 1978). Para concluir el punto, Musters agrega: [...] Se decía también que, en una ocasión en que los indios estaban acampados en el Cuheyli, o río de la ensenada de Coy, se vieron envueltos por tremendos nubarrones de denso humo negro que llegaban del oeste y que los aterrorizaron de una manera extraordinaria. Ninguna señal se halló después de pastos quemados, y se conjeturó que los indios canoeros del archipiélago Chonos habían incendiado las selvas occidentales; pero era mucho más probable que se debiera ese humo a una erupción volcánica ${ }^{2}$.

Mucho más explícita es otra mención de ese origen $y$, que ha sido conocida hace poco al encontrarse una relación que se mantenía inédita que data de 1847: Huicel, Casimiro, Centurión i muchos otros Indios que creemos dignos de fe dicen que las cordilleras terminan al sur del río Santa Cruz, i que se halla una Bahía en la última ramificación de esta con Volcán a su izquierda que hace temblar la tierra de continuo. De esta manera asentó la novedad el incógnito autor del Bosquejo sobre la Historia Natural de Magallanes $i$ las costumbres de sus habitantes (Martinic, 2001). Quien siga la relación transcrita con un mapa sectorial de Ultima Esperanza a la mano, con facilidad precisará la situación del centro volcánico, pues "la ramificación" de los Andes de que se trata debe ser identificada con la cordillera Sarmiento -el término de los Andes en la zona continental-, que para el observador distante parece hundirse en el golfo Almirante Montt (la "Bahía"), a cuya izquierda y en el fondo puede apreciarse en días despejados el monte Burney, más tarde identificado como volcán. Referencia curiosa y cabal, ciertamente.

Vida entre los Patagones (Ediciones Solar/Hachette, Buenos Aires, 1964), págs. 64 y 65.
3.- Observaciones de navegantes, viajeros, exploradores, colonos y montañeros

Esta parte es la más nutrida en referencias sobre el volcanismo en la zona andina de la Patagonia austral y la Tierra del Fuego subandina meridional, permitiendo agruparlas según la localización de los centros activos observados. Así, respecto del que por largo tiempo fue un volcán incógnito -el Lautaro-, que cuenta con el mayor número de referencias, seguido por el volcán fueguino (el antiguo San Clemente) y en menor número por las que notaron la actividad eruptiva del Reclus y del Burney. Otros centros, los volcanes Aguilera y Mimosa, tuvieron observaciones únicas.

Las referencias compulsadas acerca del cono activo andino que recibió sucesivamente las denominaciones de Chaltén, Humboldt y Lautaro, datan inicialmente de 1867 cuando el viajero inglés J.H. Gardiner y compañeros remontaban río Santa Cruz en demanda de la Cordillera; luego en 1869, la del explorador Musters, seguidas por las de Francisco P. Moreno y Carlos M. Moyano en 1876; Juan Tomás Rogers en 1877 y 1879, y Ramón Lista en 1878. Todos estos exploradores hicieron sus observaciones desde el flanco andino oriental. Por el lado occidental, esto es, navegando por los canales patagónicos, en el caso por el Messier, Thomas Brassey, en 1876, los oficiales de la corbeta norteamericana Omaha en 1878 y los del buque británico Alert, en 1879. Fueron éstos, además, quienes bautizaron Humboldt al volcán, tomándolo por una de las cumbres cimeras de la cadena andina en el sector del Campo de Hielo Patagónico Sur. Precisamente fue una carta inglesa, South American West Coast, Sheet 2 Patagonia, Magellan Strait, Gulf of Penas la que recibió esta novedad orográfica, a partir de su edición de 1881. Posteriormente el nuevo topónimo fue recogido por algunos mapas chilenos, entre ellos el tan difundido Mapa Escolar de Chile, trazado por el ingeniero José Fuenzalida y publicado en diferentes escalas en 1911 por la afamada casa alemana Justus Perthes, de Gotha. Más tarde, otro viajero, Clemente Onelli (1898) también registró la actividad del hasta entonces elusivo volcán al que ya se suponía situado en el interior del plateau andino, al noroccidente del monte Fitz Roy. Semejantes observaciones hicieron algunos colonos que desde principios del siglo XX comenzaron a poblar los campos subandinos en la vecindad del lago San Martín (después O'Higgins en la sección chilena). 
El misterio de la ubicación del centro volcánico quedó virtualmente resuelto en febrero de 1934 cuando la expedición dirigida por Federico Reichert e integrada por Ilse von Rentzell, Juan Neumayer y Arturo Donat, accedió al plateau del Campo de Hielo Patagónico Sur desde la costa del lago O'Higgins y penetró marchando hacia el sur, atraída por las emanaciones sulfurosas que traía el viento del suroeste. Los expedicionarios arribaron así al pie de un monte desconocido y pudieron observar fumarolas y notoria actividad. El hallazgo pasaría con todo inadvertido para la ciencia pues Reichert no situó geográficamente al volcán. Ignorando el descubrimiento de los alemanes, tiempo después, en 1935, el explorador Alberto De Agostini recogió nuevas evidencias de actividad (cenizas volcánicas) en el curso de uno de sus recorridos por la misma zona. Se sucedieron desde entonces nuevas observaciones ocasionales durante el cuarto de siglo siguiente, entre ellas la practicada por el glaciólogo Louis Lliboutry, quien estudiando las fotografías aéreas del área de interés (tomadas en 1944 por la Fuerza Aérea Norteamericana en convenio con el Gobierno de Chile), confirmó la efectividad de la actividad volcánica y propuso la hipótesis de dos conos activos, uno al noroeste del cerro Lautaro y otro próximo al cerro Pirámide. De ese modo se llegó al 28 de diciembre de 1959 que fue cuando se resolvió el antiguo misterio, gracias a la observación del piloto Alfonso Cuadrado, a bordo de un avión cuadrimotor Douglas DC6B de Lan Chile, realizada mientras volaba en la ruta Santiago-Puerto Montt-Punta Arenas. Avistó entonces un volcán en erupción, actividad que fotografió, registrando además la posición exacta del foco eruptivo: 49응 02'S-73을 32'O, asignándole una altura de 11.500 pies al cerro, precisando finalmente la ubicación del foco a 300 metros de la cumbre hacia el norte, situación que coincidía con la calculada años antes por Lliboutry. Arribada la nave a Punta Arenas, el piloto Cuadrado dio cuenta de la novedad al diario local La Prensa Austral, que la publicó con carácter de noticia extraordinaria ${ }^{3}$. Sería la primera noticia y la única en muchos años.

3 Ediciones de los días 28 y 29 de diciembre de 1959. El 7 de enero siguiente por nuestra parte publicamos nuestro primer artículo sobre el tema, haciendo una breve historia del volcán cuya actividad se remontaba a la memoria indígena.
Para la ciencia geográfica el descubrimiento de Cuadrado se confirmó a principios de enero de 1960 cuando el explorador británico Eric Shipton, acompañado por su compatriota Jack Ewer y por los chilenos Cedomir Marangunic y Eduardo García, ambos académicos de la Universidad de Chile, llegaron al pie del volcán, marchando de norte a sur, constatando su actividad y determinando que se trataba del cerro Lautaro, así bautizado en 1952 por Emiliano Huerta, que encabezó la primera travesía argentina completa del Campo de Hielo Patagónico Sur, de este a oeste. La confirmación de la actividad del volcán Lautaro fue comunicada al ambiente científico y recogida cartográficamente por Shipton mediante un artículo aparecido en The Geographical Journal (vol. 126, part 4, 1960) y posteriormente en su libro Land of Tempest Travels in Patagonia 1958-1962, publicado en 1963. De ese modo y al cabo de una búsqueda de un siglo cobraba vigencia geográfica el primer volcán activo de los Andes Patagónicos australes.

Recientemente ha llegado a nuestro conocimiento un nuevo antecedente. Se trata de un grabado publicado en el South American Missionary Magazine (1880). El mismo muestra en primer plano a una nave con un fondo de paisaje litoral montañoso en que destacan cumbres nevadas, de la más alta de las cuales surge una alta columna de humo blanco. El dibujo lleva por título Volcano in Smyth's Sound, Straits of Magellan viewed from H.M.S. "Gannet" y debajo una precisa leyenda complementaria: Eruptions repeated at intervals of three hours (Fig.1). La nave mencionada efectivamente pasó por el estrecho de Magallanes en 1879 comisionada, al parecer, por el Almirantazgo Británico para actuar en plan de observación de las operaciones navales del conflicto bélico que entonces enfrentaba a Chile con Perú y Bolivia. Ahora bien, la referencia de situación geográfica para la observación nos mereció inmediata duda pues no hay ni ha habido en la toponimia del Estrecho accidente alguno del nombre mencionado, que sí se da ya fuera de aquél en el afamado canal Smyth, que nace del mismo dando principio austral a la ruta marítima por el interior del Archipiélago Patagónico hasta el golfo de Penas. Ello sugería la posibilidad de que tratara del monte Burney, situado en proximidad del mencionado paso, que tiene también el carácter de centro volcánico activo. 


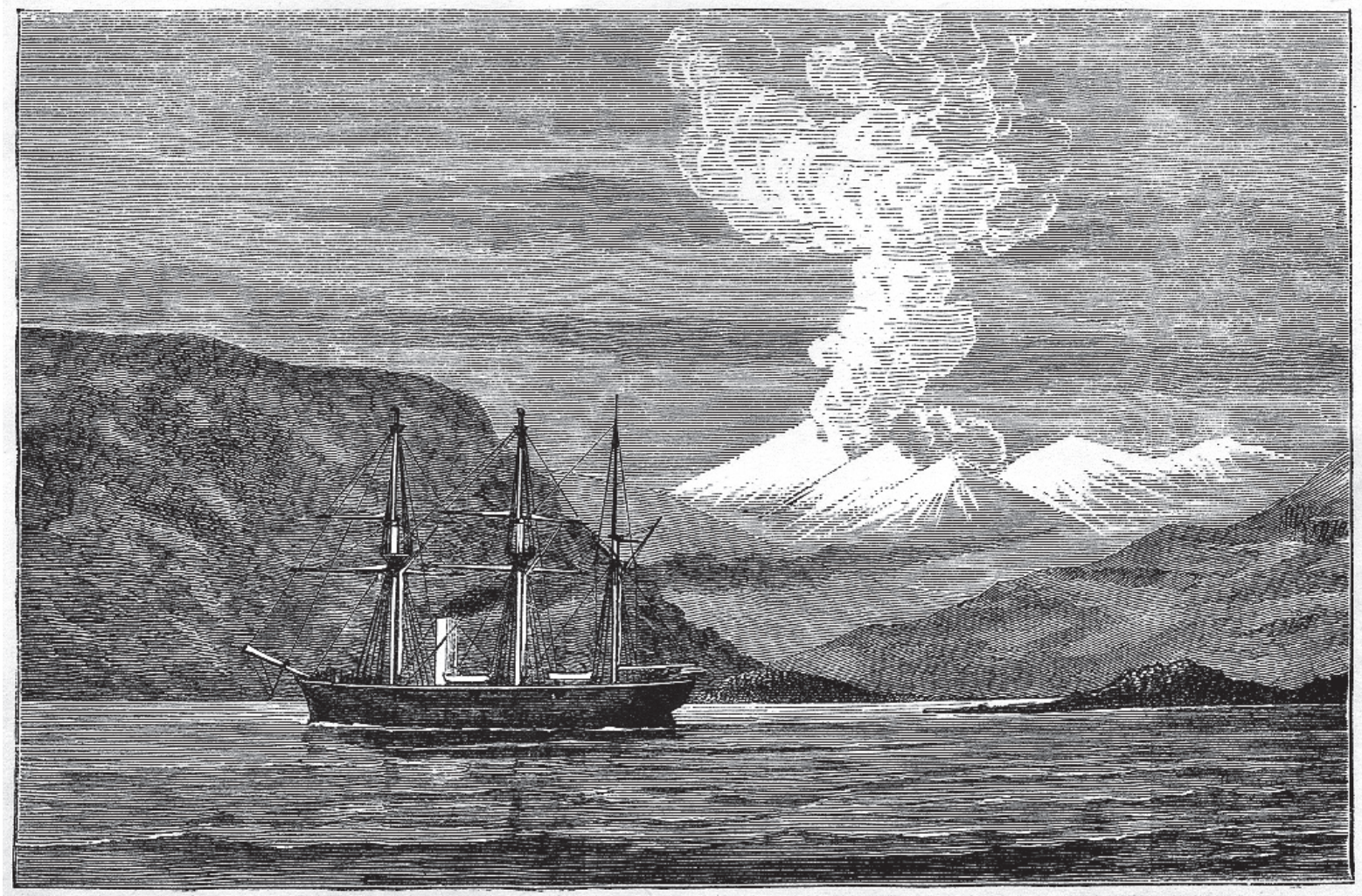

Fig. 1. Primer registro gráfico del actual volcán Lautaro en erupción, realzada desde Puerto Gray (Patagonia occidental).

En procura de la información necesaria recurrimos a la Misión Naval de Chile en Gran Bretaña y así pudimos contar con la amable colaboración del capitán de navío LT señor Otto Mrugalski, cuya búsqueda en la biblioteca del Museo Naval de Greenwich y en los Archivos Nacionales, en Richmond, resultó gratificada con el hallazgo del bitácora del H.M.S. Gannet. De su contenido nos enteramos que el día 8 de agosto de 1879 la nave se encontraba fondeada en Puerto Gray (Lat. 48응 55' S - Long. 74을 $23^{\prime} \mathrm{O}$, enfrentando por el oeste al canal Messier) y a las 11:50 horas se observó hacia el este, en las montañas de la cordillera de los Andes, una erupción volcánica que fue descrita en la forma de [...] una densa columna de vapor expulsada repentinamente del cráter de una montaña [...] que ascendió rápidamente hasta una altura de cinco o seis mil pies y adoptó una forma parecida a un hongo. Agrega la anotación que fenómenos semejantes se observaron posteriormente, mediando tres a cuatro horas entre dos de ellos. El registro de bitácora y el dibujo que dejó constancia gráfica del suceso fueron hechos en la vecindad de la isla Hume. La situación del cráter volcánico fue estimada en 49 $01^{\prime}$ de latitud sur y $73^{\circ} 30^{\prime}$ de longitud oeste (Fig. 1). Así, el grabado que se reproduce pasa a ser la primera constancia gráfica sobre la actividad del Lautaro.

Cronológicamente, el segundo centro eruptivo volcánico fue el ubicado en la sección final de los Andes Patagónicos, hacia los $51^{\circ} \mathrm{S}$. El primer registro hasta ahora compulsado data de 1879 cuando los tripulantes de la corbeta británica Alert observaron un fenómeno eruptivo en la zona andina situando el foco en la latitud $51^{\circ} 10$ 'S y denominándolo volcán Reclus. Esa única observación fue recogida en el mapa West Coast of South America from Magellan Strait to Valparaiso (Sheet 47-53ㅇ) editada en 1922 por la casa del ramo Imray, Laurie, Norie \& Wilson Ltd. Allí en un punto de la cordillera de los Andes en Ultima Esperanza, cuyas coordinadas aproximadas son 5102"S y 7330'O, se lee Active volcano registered 1879. Esta mención (como la referida al volcán Humboldt situado más al norte) había sido 
recogida por otras cartas anteriores, entre ellas el Mapa de la Rejión Austral de Chile. Provincias de Llanquihue, Chiloé i Territorio de Magallanes, de Agustín Torrealba (1904), cuya información debió provenir de cartas hidrográficas publicadas por el Almirantazgo Británico.

A base de esa única referencia, el geólogo Percy Quensel, integrante de la Expedición Sueca a la Patagonia (1907-1909), arribó en mayo de 1908 al fiordo Amalia para comprobar la vigencia del volcán, hallando efectivamente rastros de su actividad en el glaciar homónimo y en la proximidad del cerro que se eleva al oriente del mismo y al que, por su forma singular, llamó cerro Mano del Diablo atribuyéndole la condición volcánica. Tal aserto se mantuvo incuestionable por casi medio siglo hasta que el glaciólogo Lliboutry puso en duda la atribución de Quensel y acabó descartado en 1987 cuando dos montañeros franceses, Bertrand Doligez y Jean B. Hourcadette, escalaron el Mano del Diablo y comprobaron que no era un volcán. Poco después, el 21 de marzo de 1987, el geólogo Salvador M. Harambour, enterado de la observación de 1879 y de los trabajos de Quensel sobrevoló con un helicóptero la zona alto andina vecina al glaciar Amalia y encontró un foco de actividad volcánica antigua, determinando para su cráter las coordenadas 5057'50”S y 733'”O, para el que revindicó el nombre de Reclus.

El siguiente cono volcánico activo en la cadena andina de Magallanes se reveló como tal en 1910 luego que un capitán mercante que navegaba al parecer en proximidad al monte Burney, situado en la sección noroccidental de la península Muñoz Gamero, observó la montaña en erupción ${ }^{4}$. La zona fue explorada en 1962 por Eric Shipton acompañado primero por el geólogo Cedomir Marangunic, encontrándose evidencias de actividad antigua más que reciente. Luego de un intento fallido en 1963, el explorador británico acompañado por sus compatriotas Peter Radcliffe y Roger Perry consiguió alcanzar la cumbre del Burney el 10 de marzo de 1973, aunque, al parecer, sin agregar nueva información sobre su condición volcánica. Ello llama la atención toda vez que en la noche del 24 de junio de 1970

4 No hemos podido encontrar la información precisa acerca de las circunstancias de la observación ni del nombre del capitán que la hizo. Al parecer el primer autor que dio cuenta de ella fue Alberto De Agostini en su libro Andes Patagónicos (Buenos Aires, 1941).
Reinaldo y Alberto Caro, colonos radicados desde largo tiempo antes en el sector litoral del Ancón Sin salida, vieron un fogonazo en el volcán Burney y al participar el hecho a otros pobladores del sector de Puerto Ramírez, recordó el primero que hacían 50 años que ese volcán estuvo en erupción. Por su parte aquéllos sintieron un fuerte temblor hacia las veinte horas de esa misma noche, fenómeno sobre el que nada se informó entonces y que sólo llegó a ser conocido tres décadas más tarde (Martinic, 2006). Volveremos sobre el punto al referirnos a los movimientos sísmicos.

El centro volcánico del sur de la Tierra del Fuego (San Clemente, Cook) fue el primero del que se hizo registro comprobado de actividad. Ya se ha mencionado la observación original del capitán Josselin Gardin en 1712, pero sin duda la más notable fue la realizada por el capitán Basil Hall en la noche del 25 de noviembre de 1820 cuando al mando del H.M.S. Conway cruzaba desde el estrecho Le Maire hacia el cabo de Hornos. Él y su gente pudieron contemplar la erupción de un volcán, que, según lo describió después el oficial, conformó un verdadero espectáculo pirotécnico que prosiguió con intermitencias durante toda la madrugada del 26 . Hall estimó que el centro activo distaba unas cien millas hacia el interior del archipiélago fueguino. La relación que dejó el capitán inglés en un libro publicado en 1824, tuvo alguna difusión como que en 1861 la Carta Esférica del Estrecho de Magallanes preparada y publicada por el Ministerio de Marina de España, situó un volcán en las coordenadas $54^{\circ} 48^{\prime} S$ y $62^{\circ} 52$ 'O, con la leyenda Volcán visto por el Captn. Basil Hall en 1820. La confirmación de esta ubicación, tanto como de su actividad fueron a su tiempo uno de los motivos científicos de la expedición ítalo-argentina a la Tierra del Fuego en 1883, dirigida por el capitán Giovanni Roncagli, de la Marina Real de Italia, aunque nada consiguió adelantarse en la materia al punto que, según corrió el tiempo se tuvo al presunto volcán como un mito más de la geografía fueguina.

Pero, cuando nadie se acordaba del avistamiento de Hall, hacia las dos de la tarde del 3 de febrero de 1926, Emilio Krsanac, patrón de la goleta Fortunato Viejo, que navegaba por el brazo noroeste del canal Beagle en ruta de Ushuaia a Punta Arenas y, según su estimación se hallaba a unas sesenta millas del primer puerto (longitud meridiana aproximada de la isla Cook), se topó con 
una densa nube formada por lluvia fina de ceniza que se extendía por unas cinco millas y que aquél atribuyó a un volcán que podría existir en la Cordillera Darwin, a juzgar por la dirección del viento, según informó después al diario El Magallanes al arribar a Punta Arenas, medio que publicó la noticia en su edición del 18 de febrero, desde donde la exhumamos medio siglo después. Al participar más tarde este hallazgo al Dr. Manuel Suárez, del Servicio Nacional de Geología y Minería de Chile, el mismo nos informó que años antes, en 1978, una comisión geológica del Instituto de Investigaciones Geológicas (después SERNAGEOMIN) encontró casualmente domos y conos volcánicos en la isla Cook con demostraciones de actividad posteriores a la última glaciación de Fuego-Patagonia. A partir de entonces y sobre la base de los antecedentes históricos compulsados por nuestra parte, se estimó que allí estaría situada la caldera activa, a la que se denominó volcán Cook.

En 1956 ocurrió otro hecho curioso en la historia de los registros volcánicos en los Andes Patagónicos australes: un hallazgo de gabinete. Fue la observación realizada por Lliboutry al examinar las fotografías aéreas del Campo de Hielo Patagónico Sur en la zona del plateau que media entre el cordón Mariano Moreno y el monte Fitz Roy. Entonces creyó advertir evidencias en el nunatak Viedma y postuló la hipótesis de su actividad nombrándolo Volcán Viedma. Sin embargo, en 1959 llegaron al nunatak Shipton, el glaciólogo John Mercer y Geoff Bratt y advirtieron que el presunto cráter estaba conformado por rocas metamórficas, desechándose el carácter volcánico atribuido. En 1988, en una nueva aproximación al nunatak Viedma, el geólogo Rolf Kilian observó huellas de una erupción freato-magmática al sur del nunatak mismo, con lo que recobraría fuerza la hipótesis de Lliboutry.

En 1973 los montañeros británicos Leo Dickinson, Eric Jones, y Mike Coffey ascendieron el cerro que denominaron Mimosa (2.600 m), y para el que reivindicamos el antiguo de Humboldt ${ }^{5}$, situado

5 Este nombre fue tomado del velero que en 1865 transportó a los primeros inmigrantes galeses a la zona del Chubut. En vista de la ubicación del cerro en territorio chileno y de no guardar aquel suceso ninguna relación con la historia nacional, sugerimos su reemplazo toponímico por el de Humboldt en razón de su antigua vigencia cartográfica. en el término norte de la cadena del Lautaro y a unos diez kilómetros del mismo, y constataron su carácter volcánico por las fumarolas que observaron en su cima. Este hallazgo, sumado al precedente del Lautaro y a la presunta condición volcánica atribuida al nunatak Viedma, hacen posible la conjetura de existencia de una suerte de gran caldera activa subglaciar en la sección centro-norte del Campo de Hielo Patagónico Sur, circunstancia que explicaría los fenómenos repentinos de avance y retroceso de algunos glaciares, como lo han sugerido Buscaini y Metzeltin (2000:54). Es toda una apasionante incógnita para develar.

Por fin, en 1985, otro montañero británico, Matthew Hickman que lideraba una expedición chileno-británica deportiva y científica a la zona interior y periandina del fiordo Peel, que integraba además el botánico Edmundo Pisano de la Universidad de Magallanes, intentó el escalamiento del cerro Aguilera $(2.438 \mathrm{~m})$, sin conseguirlo, aunque encontró evidencias de su actividad volcánica antigua.

Con esta mención se completan las observaciones compulsadas hasta ahora acerca de la vigencia antigua o reciente de centros volcánicos en la cadena andina patagónico-fueguina (Tabla I).

¿Hay otros volcanes por descubrir en los Andes australes? Es posible, y además de la necesidad de confirmar tal condición para el Viedma, cabe recordar tan sólo la observación hecha en 1982 por uno de los montañeros de la expedición Hourcadette en el cerro que se bautizó Ecrins (2.240 m), situado en la parte superior del glaciar Dickson, quien constató la emanación de un flujo de calor en una cavidad libre, algo más abajo de la cumbre.

\section{4.- Estudios científicos}

Correspondió al geólogo finlandés Väinö Auer dar inicio a las investigaciones para determinar la actividad volcánica histórica antigua en el sur de la Patagonia, tanto en procura de la determinación cronológica de los episodios como de los focos que los habían originado. Ello ocurrió una vez que en sus trabajos encontró depósitos de ceniza en las turberas de la isla grande de Tierra del Fuego durante su expedición de 1929, a las que denominó tephras, asignándoles los números I, II y III según una antigüedad probable que estimó ser de entre 9.380 y 2.240 años antes del presente, cuya fuente atribuyó 
TABLA I. ZVA Centros volcánicos con actividad histórica reciente.

\begin{tabular}{|c|c|c|c|}
\hline Nombre & Ubicación & Carácter & $\begin{array}{c}\text { Época de observación } \\
\text { o registro }\end{array}$ \\
\hline Humboldt (Mimosa) & 4858'S/7330' aprox. & Fumarolas & 1973 \\
\hline Lautaro & $49^{\circ} 01^{\prime} \mathrm{S} / 73^{\circ} 33^{\prime} \mathrm{O}$ & Erupción, fumarolas & $\begin{array}{l}1878-79,1934-35, \\
1959-60,1995,1998\end{array}$ \\
\hline Burney & $52^{\circ} 20^{\prime} \mathrm{S} / 73^{\circ} 24^{\prime} \mathrm{O}$ & Erupción & 1910,1970 \\
\hline Cook & $54^{\circ} 57^{\prime} \mathrm{S} / 70^{\circ} 15^{\prime} \mathrm{O}$ & Erupción & $1712,1820,1926$ \\
\hline
\end{tabular}

al volcán Burney, el único centro del que él mismo, al parecer, tuvo referencia documental histórica. De estos estudios y conclusiones se enteró el ambiente científico por la publicación hecha por su compatriota Matti Salmi en 1941 (en Stern, 2008).

Tales evidencias fueron posteriormente encontradas en diferentes estratos geológicos de Patagonia y Tierra del Fuego por los norteamericanos Vera Markgraf y Calvin Heusser durante el curso de sus investigaciones paleoambientales, lo que permitió ampliar el conocimiento acerca de la amplitud geográfica del fenómeno volcánico.

Pero la mayor aproximación a la materia se tuvo con los estudios iniciados en 1974 por el vulcanólogo Charles R. Stern y proseguidos por él mismo hasta fecha reciente. Con ellos tanto consiguieron fijarse las características físico-químicas del material volcánico depositado en diferentes yacimientos, determinar su procedencia de distintos focos activos y establecer una tefrocronología válida para los fenómenos vulcanológicos de Sud-Patagonia durante un rango temporal extendido entre 14.000 y 3.000 años antes del presente. Al mismo se debe, además, la sectorización geográfica de los conos eruptivos con su concepto de Zona Volcánica Austral (AVZ en inglés). De todos estos estudios y conclusiones se ha dado cuenta a la comunidad científica (Stern 1976, 1982, 1984, 1990, 1991, 1992, 2004, 2008 y en Stern y Kilian, 1996).

Estos estudios fueron en parte sincrónicos y nutridos además con los palinológicos y paleoambientales de Markgraf, Heusser y otros investigadores, y en especial con los trabajos arqueológicos iniciados por Junius Bird en 1936-37 en la zona centro-oriental magallánica, y con los desarrollados por Hugo Nami, Alfredo Prieto y Mauricio Massone desde mediados de los años de 1980 en diferentes yacimientos de Ultima Esperanza y Tierra del Fuego. Los mismos pusieron en evidencia, confirmando lo revelado en el transcurso de los años de 1930 y 1950 para la zona oriental de Magallanes por Bird, Emperaire y Laming (Cuevas Fell, Pali Aike y otras), que el volcanismo fue un fenómeno que aunque aperiódico fue recurrente y con efectos catastróficos para la existencia de los grupos humanos de Patagonia continental $e$ incluso para la supervivencia de especies animales pleistocénicas tardías, llegando a generar intervalos prolongados de interrupción o alteración en la vigencia de los mismos.

Los estudios de Stern han sido complementados en época reciente por los trabajos de Rolf Kilian, que se han centrado de preferencia en la península Muñoz Gamero (Gran Campo Nevado), que han permitido extender la actividad del volcán Burney hasta bien avanzado el Holoceno (ca. 1.9001.500 A.P.).

Esta síntesis da cuenta del notable grado de adelanto logrado en el conocimiento respecto de una materia por demás interesante, tocante a la cual cabe esperar nuevos aportes en el próximo futuro (Tabla II y Fig. 2).

\section{II.- ACTIVIDAD SISMICA}

En la madrugada (2 horas 55 minutos) del 17 de diciembre de 1949 los habitantes de Punta Arenas y los sectores cercanos de la zona central de Magallanes fueron sorprendidos por un movimiento sísmico con carácter de terremoto que tuvo numerosas réplicas en el transcurso del día, entre ellas la más fuerte registrada a las 11:10 de la mañana, que fue el movimiento de mayor intensidad (estimado en grado 8 de la escala Richter). El fenómeno fue percibido también en el faro San Isidro y en la bahía San Nicolás (suroeste de la península de Brunswick), lugar en el que, según se supo después ocasionó derrumbes que causaron la muerte de tres personas; en Caleta María (fiordo del Almirantazgo), en Porvenir (Tierra del Fuego), en cuyo sector litoral aledaño de Punta Chilota se observó un inusual 
TABLA II. ZVA Centros volcánicos con actividad histórica antigua.

\begin{tabular}{|c|c|c|c|}
\hline Nombre & Ubicación & Carácter & Vigencia aproximada \\
\hline Aguilera & $50^{\circ} 20^{\prime} \mathrm{S} / 73^{\circ} 45^{\prime} \mathrm{O}$ & Erupción & circa 6.300 y 3.345 A.P. \\
\hline Reclus & $50 \div 57^{\prime} 50 " \mathrm{~S} / 73^{\circ} 35^{\prime} 5^{\prime \prime O}$ & Erupción & circa 14.990 y 3.345 A.P. \\
\hline Burney & $52^{\circ} 20^{\prime} \mathrm{S} / 73^{\circ} 244^{\prime} \mathrm{O}$ & Erupción & circa 3.970 y 1.500 A.P. \\
\hline Diablo & $52^{\circ} 07^{\prime} \mathrm{S} / 69^{\circ} 35^{\prime} \mathrm{O}$ aprox. & Erupción & menor a 15.000 A.P. \\
\hline
\end{tabular}

fuerte oleaje a modo de tsunami, según el diario El Magallanes; en Punta Delgada y en Río Gallegos hacia el oriente del probable epicentro. Amén de sorpresiva, desagradable y lamentable por alguna de sus consecuencias, la experiencia parecía no tener precedente histórico aunque quizá alguno muy anciano entre sus vecinos pudo recordar una situación semejante durante su niñez.

En efecto, setenta años antes, un movimiento sísmico de semejante violencia se había registrado a las 3 horas 30 minutos del 1ํ de febrero de 1879 . Su fuerza expansiva y su extensión geográfica han podido ser determinadas con nuestra compulsa de anotaciones referidas al fenómeno, que permitieron establecer que el mismo fue percibido prácticamente en simultaneidad en diferentes lugares de la Patagonia austral y la Tierra del Fuego: en Punta Arenas, con sorpresa y alarma para sus habitantes; hacia el norte y noreste de la Colonia, en la zona de Laguna Blanca por lady Florence Dixie y compañeros; en el lago Argentino por Juan Tomás Rogers; hacia el oeste, en el mar de Skyring, por los tripulantes de la corbeta Magallanes y en el sector occidental del estrecho de Magallanes (Puerto Gallant), por los tripulantes del vapor de guerra francés La Magicienne; hacia el este, en la zona atlántica de la Tierra del Fuego por Ramón Serrano Montaner y compañeros, y hacia el sur de la misma isla, en la Misión de Ushuaia, por el pastor Thomas Bridges. Ello representa un rango longitudinal de no menos de 600 kilómetros y latitudinal de unos 400 kilómetros.

Por tratarse de la primera experiencia de gente civilizada en el meridión chileno es de interés conocer la relación del antiguo Gobernador de Magallanes, Diego Dublé Almeida, acerca de la impresión colectiva por el movimiento sísmico, que lo sorprendió de paso por Punta Arenas: El 1o. de Febrero, a las 3.40 a.m., hubo en Punta Arenas un fuerte temblor que duró más de un minuto y que merece el nombre de terremoto. No causó daño ninguno en los edificios porque son todos de madera y de un piso. El espanto en la población fue extraordinario. Aquí jamás había temblado y todos temían algún cataclismo. Ha continuado temblando por tres días, pero los estremecimientos de la tierra no han sido tan fuertes como el primero. Los tripulantes de la "Magicienne", buque de guerra francés que se hallaba al ancla en Puerto Cumberland ${ }^{6}$, en el Estrecho, sintieron el gran temblor. Todos a bordo creyeron que alguna explosión había ocurrido en la máquina del buque, tal fue el ruido que produjo?.

Aunque ni en uno ni otro caso pudo de momento determinarse, el posible epicentro por falta absoluta de conocimiento sobre la sismicidad en el territorio magallánico, sí pudo hacerse muchos años después al establecerse que en ambos casos el mismo había estado en un sector de la zona centro occidental del estrecho de Magallanes que coincidía, en general, con la orientación noroeste-sureste del gran canal interoceánico y su prolongación en la última dirección señalada por los canales Gabriel y Cascada que separan la isla Dawson de la Tierra del Fuego, por el fiordo del Almirantazgo, el valle del río Azopardo desaguadero del lago Fagnano y la cuenca lacustre correspondiente en la isla grande de Tierra del Fuego. Había sido precisamente en alguno de los sectores ribereños o litorales donde se habían registrado derrumbes y habían fallecido personas durante el terremoto de 1949.

Se sabe igualmente de antiguos movimientos de origen tectónico por la arqueología. En efecto, los trabajos de Junius Bird durante la década de 1930, proseguidos durante los años de 1980 y 1990 por Hugo Nami, Alfredo Prieto y Mauricio Massone en diferentes yacimientos, han puesto en evidencia la ocurrencia de tales fenómenos de los

6 Este topónimo no es conocido para el Estrecho, por lo que creemos que se trata de un error de Dublé. Sí consta, en cambio la permanencia de la nave en Puerto Gallant (península de Brunswick) en la fecha del fenómeno de que se trata.

7 Diario de Viaje al Río Santa Cruz, Patagonia, (Revista Chilena de Historia y Geografía, №93, Santiago, 1938), pág. 279. 
que no ha podido determinarse si fueron sincrónicos o sucesivos. La época probable de tales fenómenos ha sido situada entre 12.600 y 9.000 años antes del presente. Al respecto, cabe una comparación entre el movimiento de 1949, tenido como el más fuerte hasta ahora registrado y que sin embargo no ocasionó derrumbe alguno importante en la Cueva del Milodón, y aquellos que debieron darse en el remoto pasado y que provocaron la caída de grandes bloques en ese sitio tan conocido, en la Cueva del Medio, en la Cueva 1 del Lago Sofía, en la Cueva Fell y en las de Los Toldos y Las Buitreras, estas últimas situadas en Santa Cruz (Argentina), sellando inclusive ocupaciones humanas preexistentes. Por tanto debería aceptarse que en tales movimientos sísmicos la intensidad debió superar, por sus efectos, los 8 grados de la escala de Richter estimados en 1949. De su ocasional repetición en el tiempo

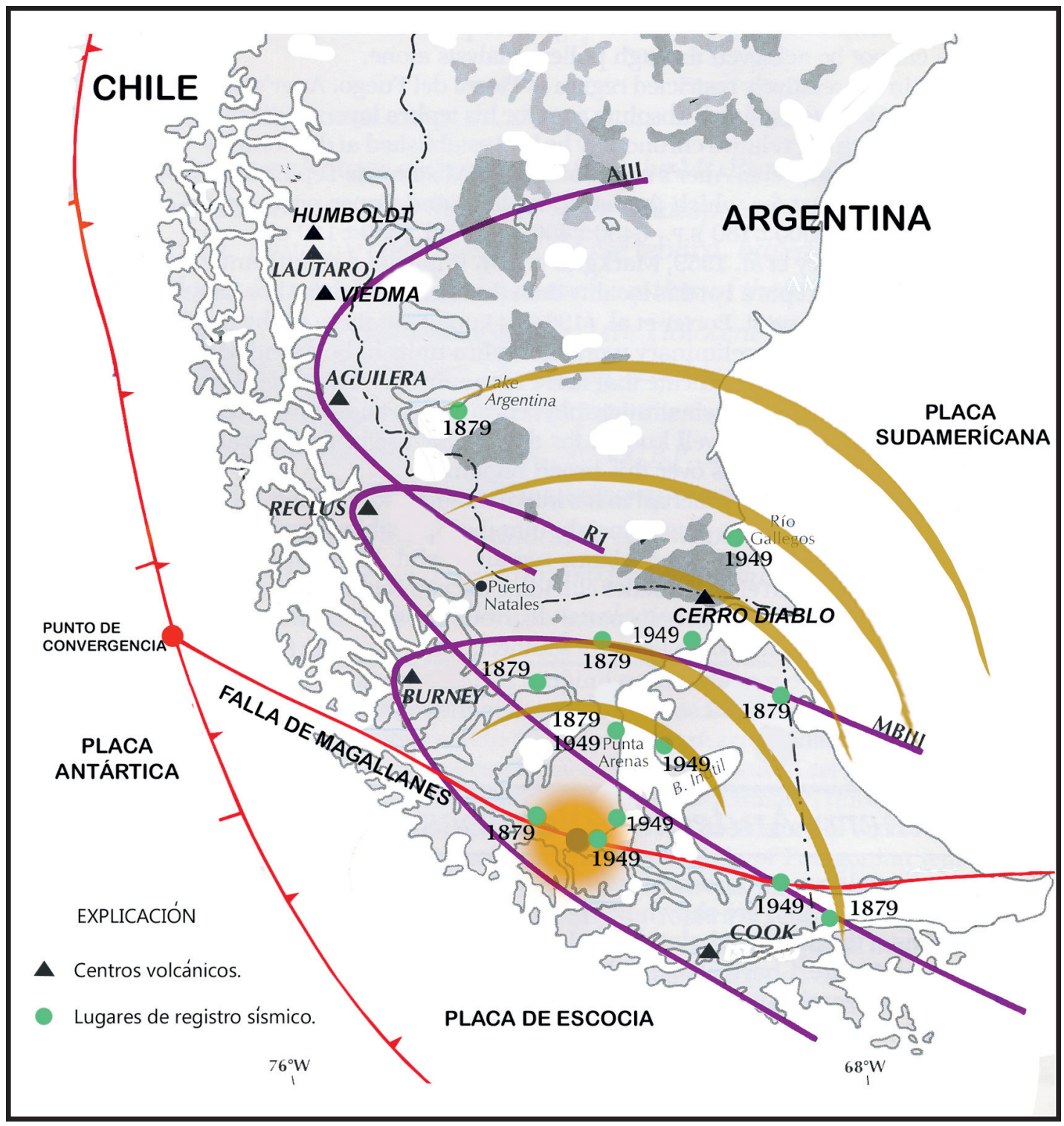

Fig. 2. Zonas Volcánica y Sísmica Australes (Adaptado y modificado de Stern 1990, 2008; Cisternas y Vera 2008).

NOTA: Las líneas elípticas señalan las áreas de extensión de la ceniza volcánica de acuerdo con el estudio de Stern; las líneas que indican la expansión circular dan cuenta de los registros de los movimientos sísmicos de 1879 y 1949. 
histórico da cuenta la tradición aónikenk recogida por Musters, según se ha visto antes al tratar del volcanismo.

Estudios realizados a contar de mediados de los años de 1970 permitieron hacer luz acerca de algunos aspectos fundamentales de la geología estructural de Magallanes, entre ellos la existencia de la Falla del mismo nombre, coincidente en su ubicación con la orientación marítimo-fluvio-lacustre mencionada, que señala la separación de las placas tectónicas Sudamericana y Antártica, situándose inclusive al noroeste de los islotes Evangelistas el punto triple de convergencia entre las dos placas mencionadas y una tercera, la de Scotia.

El trabajo más completo y reciente sobre la materia se debe al ingeniero Rodrigo Adaros, y permite concluir que la sismicidad se origina en el roce de las placas Sudamericana y Antártica, con mayor intensidad al norte que al sur de la Falla de Magallanes (Adaros, 2003). He aquí la explicación para los movimientos sísmicos históricos mencionados $\mathrm{y}$, posiblemente, para otros sucesos de la especie de los que dejó constancia Lautaro Navarro Avaria $^{8}$ (Fig. 2).

Además de estos movimientos originados en la sismicidad tectónica, hay constancia de otros debidos al volcanismo, aspecto sobre el que casi nada se conoce. A esta especie pertenece el temblor ocurrido en la noche del 24 de junio de 1970, percibido por colonos de Puerto Ramírez y Ancón Sin Salida, al que se ha hecho referencia anterior, que se dio en contemporaneidad con una erupción del volcán Burney (Martinic, 2006). Al mismo origen, por fin, deben atribuirse los temblores menores percibidos ocasionalmente en Puerto Natales y alrededores durante los años de 1980 y 1990 , según informaciones de prensa, cuyos focos podrían ser situados en el monte Burney como en el volcán Reclus, no obstante que a éste se le tiene por extinguido9. Precisamente, una de las comprobaciones más interesantes del estudio desarrollado por Adaros es la referida a la

8 Censo General del Territorio de Magallanes (Punta Arenas, 1908), tomo II, págs. 498 y 499.

9 Recientemente se han observado señales de actividad, de acuerdo con las declaraciones de Camilo Rada, un montañero que ascendió hasta el cráter del Reclus (Declaraciones al diario La Prensa Austral, edición de fecha 9 de mayo de 2008, y además en sitio www.expenews.or). sismicidad activa de origen volcánico, en el caso asociada al volcán Reclus según los registros de monitoreo obtenidos para los meses de abril y mayo de 1998.

Nada puede resultar más engañoso, bien se sabe, que el sueño aparente de los volcanes. De allí que es aconsejable el monitoreo periódico de los focos volcánicos del Reclus y del Burney, como de otros centros de actividad comprobada si fuera necesario. En este respecto, debe destacarse la iniciativa de Rodrigo Adaros y su desarrollo en forma del Experimento Sísmico en la Patagonia y Antártica (SEPA) durante 1997 y 1998, en el que participaron la Universidad de Chile y Washington University (Saint Louis, MO, EE.UU. de América), que permitió la instalación de ocho estaciones sismográficas desde el sur de Patagonia hasta la Península Antártica. De ellas se mantiene operativa hasta la actualidad la de Puerto del Hambre, ubicada medio centenar de kilómetros al sur de Punta Arenas (Lat. 53은's; long. 70: 55’0).

La conclusión principal que extraemos del estudio mencionado es que sin embargo de haberse considerado a priori la macro región austral americana y chilena como una zona de baja sismicidad, es de gran interés científico la prosecución de los estudios en ella, por cuanto la concurrencia en la misma de tres placas tectónicas conforma un hecho geológico excepcional que así lo amerita.

\section{CONCLUSIONES}

Finalmente cabe convenir en que el registro histórico de los fenómenos de que se da cuenta es ciertamente conveniente porque contribuye a la mejor información de la comunidad científica especializada en su estudio con noticias basadas en la experiencia de los habitantes, que inclusive ha podido ser de consecuencias traumáticas para ellos y catastróficas para el ambiente natural, y cuya repetición podría prevenirse y mitigarse en sus eventuales efectos, con una adecuada difusión a la comunidad.

La experiencia reciente en las zonas norte y central de la Patagonia chilena demuestra cuán errada puede ser la creencia común de que los centros volcánicos inactivos desde tiempo inmemorial habrán de permanecer en tal situación para siempre. Los casos de lo acontecido con los volcanes Hudson y Chaitén son pruebas elocuentes de lo contrario. 
Del mismo modo creer que la Región Magallánica se encuentra libre de convulsiones telúricas en forma de temblores o terremotos, formando como forma un distrito particular donde confluyen e interactúan tres placas tectónicas. Conocer lo acontecido históricamente en uno y otro aspecto y extraer enseñanzas de las experiencias perjudiciales para la vida humana, la economía y la naturaleza, ciertamente que ayudará a prevenir o a morigerar las eventuales consecuencias en caso de repetición ocasional de los fenómenos descritos.

\section{AGRADECIMIENTOS}

Agradecemos al señor Denis Chevallay la referencia sobre la figura 1 incluida en el artículo, así como al capitán de navío LT señor Otto Mrugalski, de la Misión Naval de Chile en Gran Bretaña, por la información brindada para precisar el sitio en que se realizó el dibujo de la misma.

\section{FUENTES DE CONSULTA}

ADAROS C., RODRIGO E. 2003 Sismicidad y Tectónica del Extremo Sur de Chile. Tesis para optar al grado de Magister en Ciencias Mención Geofísica. Universidad de Chile. Santiago.

BIRD, JUNIUS B. (1988) 1993 Viajes y arqueología en Chile Austral. Ediciones de la Universidad de Magallanes. Punta Arenas.

BUSCAINI, GINO y SILVIA METZELTIN 2000 Patagonia. Tierra mágica para viajeros y alpinistas. Desnivel Ediciones, Barcelona.

CASASSA, GINO y ANDRES RIVERA 2001 Campo de Hielo Patagónico Sur: Su historia. Anales del Instituto de la Patagonia Serie Ciencias Humanas, volumen 29:2978. Punta Arenas.

CISTERNAS, ARMANDO y EMILIO VERA 2008 Sismos históricos y recientes en Magallanes. Magallania, volumen 36, No. 1: 43-51.Punta Arenas.

DUBLE ALMEIDA, DIEGO 1938 Diario de viaje al río Santa Cruz, Patagonia. Revista Chilena de Historia y Geografía № 93. Santiago.

HARAMBOUR, SALVADOR M. 1988 Sobre el hallazgo del mítico volcán Reclus, ex Mano del Diablo, Hielo Patagónico Sur, Magallanes. Revista Geológica de Chile, volumen 15, No. 2:173-179. Santiago.

LLIBOUTRY, LOUIS 1956 Nieves y Glaciares de Chile. Ediciones de la Universidad de Chile. Santiago.
MARTINIC B. MATEO 1960a El misterioso volcán de la Patagonia. La Prensa Austral, 7 de enero de 1960. Punta Arenas.

-1960b. Descubrimiento de un nuevo volcán en la Patagonia Revista Geográfica de Chile Terra Australis No. 18:188-192. Santiago.

-1982. Hielo Patagónico Sur. Publicaciones del Instituto de la Patagonia. Punta Arenas.

-1985. El volcán fueguino del capitán Hall. Revista Patagónica No. 22:5-8. Buenos Aires.

-1988a. El gran temblor de tierra de 1879 en la Patagonia austral. Revista Patagónica No. 37:30-31. Buenos Aires.

-1988b. Actividad volcánica histórica en la Región de Magallanes. Revista Geológica de Chile, volumen 15; No.2:181-186. Santiago.

-1997. Rarezas cartográficas: I Las cuatro versiones del Mapa de Chile del Padre Alonso de Ovalle. II El curioso Mapa de la Región Magallánica de Francisco Seixas y Lovera (1690). Boletín de la Academia Chilena de la Historia No. 107:385-400. Santiago.

-1999. Cartografía Magallánica 1523-1945. Ediciones de la Universidad de Maga1lanes. Punta Arenas.

-2001. Documentos inéditos para la Historia de Magallanes. Anales del Instituto de la Patagonia Serie Ciencias Humanas, volumen 29:211-238. Punta Arenas

-2006. Documentos inéditos para la Historia de Magallanes: El fallido intento colonizador en Muñoz Gamero (1969-1971). Magallania vol.34(2): 119-124.

-2007. Los Césares de la Patagonia ¿Otra fuente indígena para la leyenda, o una hasta ahora desconocida fuente creación del imaginario aónikenk? Magallania, volumen 35 No. 2:7-14. Punta Arenas.

MASSONE, MAURICIO 1987 Los cazadores paleo-indios de Tres Arroyos (Tierra del Fuego). Anales del Instituto de la Patagonia, Serie Ciencias Sociales, Volumen 17:47-60. Punta Arenas.

METZELTIN SILVIA y GINO BUSCAINI 1999 Volcanes Australes. Cuadernos Patagónicos No. 15. TECHINT. Buenos Aires.

NAMI, HUGO 1987 Cueva del Medio: perspectivas arqueológicas para la Patagonia Austral. Anales del Instituto de la Patagonia, Serie Ciencias Sociales, volumen 17:73-106. Punta Arenas.

PRIETO, ALFREDO 1991 Cazadores tempranos y tardíos en la Cueva 1 del lago Sofía. Anales del Instituto de la Patagonia, Serie Ciencias Humanas, volumen 20:6785, Punta Arenas. 
SHIPTON, ERIC 1963 Land of Tempest. Travels in Patagonia 1958-62. Hodder and Stoughton. London.

SKEWES, MILKA 1978 Geología, petrología, quimismo y origen de los volcanes del área de Pali-Aike, Magallanes Chile. Anales del Instituto de la Patagonia, volumen 9:95-106. Punta Arenas.

STERN, CHARLES 1990 Tephrochronology of Southernmost Patagonia. National Geographic Research, volumen six, pp. 110-126. Washington,

-1992. Tefrocronología de Magallanes: nuevos datos e implicaciones. Anales del Instituto de la Patagonia, Serie Ciencias Humanas, volumen 21:129-141. Punta Arenas.

-2008. Holocene tephrochronology record of large explsive eruptions in the Southernmost Patagonian Andes. Bulletin of Vulcanology. vol. 70, p. 435-454.

STERN, CHARLES y ROLF KILIAN 1996 Role of subducted slab, mantle wedge and co continental crust in the generation of adakitic andesites from the Austral Volcanic Zone, southernmost Andes, Contributions to Mineralogy and Petrology, v. 123, p. 263-281.
STERN, CHARLES, ALEXANDRA SKEWES Y MANUEL DURAN 1976. Volcanismo orogénico en Chile austral. Actas Primer Congreso Geologico Chileno, Santiago, Chile, v. 2, p. 195-212.

STERN, CHARLES, KYOTO FUTA Y KARLIS MUEHLENBACHS 1984. Isotope and trace element data for the orogenic andesites from the austral Andes. In Andean Magmatism: Chemical and Isotopic Constraints (R. Harmon and B. Barreiro, editors), Shiva Press, Ltd., Chesire, England, p. 31-46.

STERN, CHARLES AND 6 OTHERS 2007. Chilean Volcanoes. Chapter 5 in The Geology of Chile (T Moreno \& W. Gibbons, eds), Geologic Society of London, p. 149-180

SUAREZ, MANUEL 1976 La Cordillera Patagónica: su división y relación con la Península Antártica. Anales del Instituto de la Patagonia, volumen 7:105-113. Punta Arenas. 